\title{
Hydrodynamic excitations of Bose Condensates in anisotropic traps
}

\author{
Martin Fliesser ${ }^{1}$, András Csordás ${ }^{2}$, Péter Szépfalusy ${ }^{3}$, Robert Graham ${ }^{1}$ \\ ${ }^{1}$ Fachbereich Physik, Universität-Gesamthochschule Essen, 45117 Essen, Germany \\ ${ }^{2}$ Research Group for Statistical Physics of the Hungarian Academy of Sciences, \\ Múzeum krt. 6-8, H-1088 Budapest, Hungary \\ ${ }^{3}$ Institute for Solid State Physics, Eötvös University, Múzeum krt. 6-8, H-1088 Budapest, Hungary, and \\ Research Institute for Solid State Physics, P.O. Box 49, H-1525 Budapest, Hungary,
}

(September 17, 2018)

\begin{abstract}
The collective excitations of Bose condensates in anisotropic axially symmetric harmonic traps are investigated in the hydrodynamic and Thomas-Fermi limit. We identify an additional conserved quantity, besides the axial angular momentum and the total energy, and separate the wave equation in elliptic coordinates. The solution is thereby reduced to the algebraic problem of diagonalizing finite dimensional matrices. The classical quasi-particle dynamics in the local density approximation for energies of the order of the chemical potential is shown to be chaotic.
\end{abstract}

03.75.Fi,05.30.Jp,32.80.Pj,67.90.+z

The new Bose condensates of alkali atoms in magnetic traps [1] 3] offer a unique way to investigate the low-lying collective excitations in Bose condensates [4 16]. Experimentally collective modes with a given symmetry have been excited by time-dependent modulations of the trapping potential, and their evolution has been followed in real time by measurements of the resulting shape oscillations of the condensates. The measurements performed so far have involved turning off the trap after a given time [4] 6], but in the future they could even be performed non-destructively by elastic off-resonant light scattering [7]. Theoretically the collective modes have been analyzed by using the Bogoliubov-equations or by linearizing the time-dependent Gross-Pitaevskii equation around the time-independent condensate and solving these equations numerically [8,9] or analytically in various approximations [10 16]. Very good agreement between the numerical and the experimental results has been found.

In a seminal paper Stringari [16] has shown how the coupled wave equations for the collective excitations are simplified in the hydrodynamic limit to become a single second-order wave equation for density waves, and he obtained analytical solutions for all its modes in spherically symmetric harmonic traps, and, remarkably, also for some of its modes in axially symmetric harmonic traps. The latter are particularly important, because all experiments have been performed with traps of this symmetry $\left[\begin{array}{ll}6 & 6\end{array}\right.$.

In the present paper it is our goal to study in more detail by analytical means the hydrodynamic wave equation in the axially symmetric case. We wish to find an explanation why at least some analytical solutions have been possible in this case and intend to use this insight to construct more solutions in a systematic way.

In principle the collective mode problem looks very different for isotropic and for axially symmetric traps: In the isotropic case the rotational symmetry ensures that angular momentum conservation gives two good quan- tum numbers, and therefore the wave equation is separable in spherical coordinates. For axial symmetry, however, only the axial component of angular momentum remains a good quantum number, besides the energy, and one may expect that the system, having three degrees of freedom, is not integrable. In fact, this expectation is born out for collective excitations whose energies are neither very large nor very small compared to the chemical potential (see below), which spoils all hopes to find exact analytical solutions for the modes and their spectrum on this energy range for axially symmetric traps. Why then are such solutions possible at energies in the hydrodynamic regime, i.e. for energies much smaller than the chemical potential?

The answer is provided by the existence, in that regime, of an additional conserved quantity which we exhibit explicitely below. Its existence permits the separation of the hydrodynamic wave equation in elliptical coordinates. Thereby the task of solving the wave equation can be reduced to the purely algebraic problem of diagonalizing finite dimensional matrices. We use this method to obtain the spectrum of the low lying hydrodynamic modes as a function of the ratio of the axial and the radial trap frequencies.

A convenient starting point of our analysis are the linearized hydrodynamic equations as derived in 16]. They read

$$
\dot{\varphi}=-\frac{4 \pi \hbar a}{M} \delta \rho, \quad \delta \dot{\rho}=-\frac{\hbar}{M} \boldsymbol{\nabla} \cdot \rho_{0}(\boldsymbol{x}) \boldsymbol{\nabla} \varphi
$$

Here $\varphi$ is the phase of the macroscopic wave function, $\delta \rho$ the local perturbation of the number density in the collective mode, $\rho_{0}(\boldsymbol{x})$ is the number density of the timeindependent condensate in the Thomas-Fermi approximation [18], $\rho_{0}(\boldsymbol{x})=\frac{M}{4 \pi \hbar^{2} a}(\mu-U(\boldsymbol{x})) \Theta(\mu-U(\boldsymbol{x}))$, where $\mu$ is the chemical potential, $M$ and $a$ are the mass and the positive $s$-wave scattering length of the atoms. For the connection of (11) with the Bogoliubov-equations see 
[17. The trapping potential $U(\boldsymbol{x})$ is assumed to have the form $U(\boldsymbol{x})=(M / 2) \omega_{0}^{2}\left(x^{2}+y^{2}\right)+(M / 2) \omega_{z}^{2} z^{2}$. The surface of the condensate, in the Thomas-Fermi limit is defined by $U=\mu$. Eqs. (1) are obtained by a gradient expansion and assuming $\delta \rho$ and $\nabla \varphi$ to be small. Eliminating $\boldsymbol{\nabla} \varphi$ from both equations and making the ansatz $\delta \rho(\boldsymbol{x}, t)=e^{-i \omega t} \psi(\boldsymbol{x})$ one obtains the time-independent wave equation [16] $M \omega^{2} \psi(\boldsymbol{x})=-\boldsymbol{\nabla} \cdot(\mu-U(\boldsymbol{x})) \boldsymbol{\nabla} \psi(\boldsymbol{x})$ which holds inside the condensate. We look for boundary conditions on the surface of the condensate which make the operator $\hat{G}=-M^{-1} \boldsymbol{\nabla} \cdot(\mu-U(\boldsymbol{x})) \boldsymbol{\nabla}$ Hermitian, so that for two eigenfunctions $\psi, \tilde{\psi}$ with eigenvalues $\omega^{2}, \tilde{\omega}^{2}$ we have orthogonality according to

$$
\begin{aligned}
& \left(\omega^{2}-\tilde{\omega}^{2}\right) \int_{V} d^{3} x \psi^{*} \tilde{\psi}=\int_{V} d^{3} x\left[\psi^{*} \hat{G} \tilde{\psi}-\tilde{\psi} \hat{G}^{*} \psi^{*}\right] \\
& \quad=-M^{-1} \int_{\partial V} d f(\mu-U)\left(\psi^{*} \partial \tilde{\psi} / \partial n-\tilde{\psi} \partial \psi^{*} / \partial n\right)=0 .
\end{aligned}
$$

Here $V$ and $\partial V$ denote the volume of the condensate and its surface, respectively. Because $\mu-U$ vanishes on the boundary it is enough to require that $\psi$ and its normal derivative $\partial \psi / \partial n$ remain bounded there. In the following it will be useful to measure lengths in units of the radial Thomas-Fermi radius $r_{0}=\left(2 \mu / M \omega_{0}^{2}\right)^{1 / 2}$, which brings the wave equation into the dimensionless form $\omega^{2} \psi=\hat{G} \psi$ with

$$
\hat{G}=-\left(\omega_{z}^{2} / 2\right) \boldsymbol{\nabla} \cdot\left(\left(1-\epsilon^{2}\right)\left(1-\rho^{2}\right)-z^{2}\right) \boldsymbol{\nabla}
$$

where we use cylindrical coordinates $\rho=\sqrt{x^{2}+y^{2}}, z$ and the azimuthal angle $\phi$ and define $\epsilon^{2}=1-\omega_{0}^{2} / \omega_{z}^{2}$, which is positive for $\omega_{0}<\omega_{z}$. The operator $\hat{G}$ commutes, of course, with the axial angular momentum operator $\hat{L}_{z}=-i \frac{\partial}{\partial \phi}$. However, there is an additional nontrivial operator

$$
\hat{B}=-\boldsymbol{\nabla}^{2}+(\boldsymbol{x} \cdot \boldsymbol{\nabla})^{2}+3 \boldsymbol{x} \cdot \boldsymbol{\nabla}+\epsilon^{2} \partial^{2} / \partial z^{2}
$$

which commutes with $\hat{L}_{z}$ and with $\hat{G}$, as one may check by direct calculation of the commutators. In the isotropic case $\epsilon=0, \hat{B}$ may be expressed by the square of the angular momentum $\hat{L}^{2}=\hat{L}_{x}^{2}+\hat{L}_{y}^{2}+\hat{L}_{z}^{2}$ and $\hat{G}$ via $\hat{B}=\hat{G} / \omega_{0}^{2}+\hat{L}^{2}$. Because of the existence of the three commuting operators $\hat{G}, L_{z}$, and $\hat{B}$ in the system with the three degrees of freedom $\rho, z, \phi$ it is now manifest that the system is integrable, i.e. the eigenvalues of $\hat{G}$ can be labelled by the quantum numbers of $L_{z}$ and $\hat{B}$. To see this explicitely we now look for variables in which the wave equation separates and introduce cylindrical elliptical coordinates $\xi, \eta$, which in their oblate spheroidal form [19] are defined by $\rho=\sigma \sqrt{\left(\xi^{2}+1\right)\left(1-\eta^{2}\right)}, \quad z=\sigma \xi \eta$. These coordinates are orthogonal. Surfaces of $\xi=$ const are confocal ellipsoids with foci at $z=0, \rho=\sigma$. Surfaces with constant $\eta$ are confocal hyperboloids with the same foci. For $\omega_{z} \geq \omega_{0}$, i.e. $0 \leq \epsilon^{2} \leq 1$, the foci at $z=0$ $\rho=\sigma$ are made to coincide with the foci of the ellipsoidal
Thomas-Fermi surface if we choose $\sigma=\epsilon$. The ThomasFermi surface is given by $\xi_{T F}=\left(1 / \epsilon^{2}-1\right)^{1 / 2}$. Then the interior of the condensate is described by $\xi$ in the range $\left[0, \xi_{T F}\right]$ and $\eta$ in the range $[-1,1]$.

For $\omega_{z} \leq \omega_{0}$ the parameter $\epsilon^{2}$ is no longer usefull as it becomes negative. Instead one can define $\epsilon^{\prime 2}=1-$ $\omega_{z}^{2} / \omega_{0}^{2}=-\epsilon^{2} /\left(1-\epsilon^{2}\right)$ which lies in the range $0 \leq \epsilon^{\prime 2}<$ 1. The foci of the Thomas-Fermi ellipse now lie at $z=$ $\pm \sigma^{\prime}$ where $\sigma^{\prime}=\epsilon^{\prime} / \sqrt{1-\epsilon^{\prime 2}}$. Therefore we now need the prolate spheroidal form of elliptical coordinates [19] with foci at $z= \pm \sigma^{\prime}, \rho=0$. These coordinates are defined by $\rho=\sigma^{\prime} \sqrt{\left(\xi^{2}-1\right)\left(1-\eta^{2}\right)}, \quad z=\sigma^{\prime} \xi \eta$ where inside the condensate $\xi$ now has the range $\left[1,1 / \epsilon^{\prime}\right]$ while $\eta$ has the same range as before. The treatments in the two cases are equivalent via the transformation connecting $\epsilon$ and $\epsilon^{\prime}$. In the following we shall present the equations for the case $\omega_{z}>\omega_{0}$. The final formulas for $\omega^{2}$ apply for $\omega_{z} \geq \omega_{0}$ and $\omega_{z} \leq \omega_{0}$. After the change of coordinates with $\sigma=\epsilon$ the operator $\hat{G}$ takes the form

$$
\begin{gathered}
\hat{G}=\frac{\omega_{z}^{2}}{2 \epsilon^{2}} \frac{1}{\xi^{2}+\eta^{2}} \\
{\left[\left(1-\epsilon^{2}\left(1-\eta^{2}\right)\right) \frac{\partial}{\partial \xi}\left(1-\epsilon^{2}\left(\xi^{2}+1\right)\right)\left(\xi^{2}+1\right) \frac{\partial}{\partial \xi}\right.} \\
+\left(1-\epsilon^{2}\left(\xi^{2}+1\right)\right) \frac{\partial}{\partial \eta}\left(1-\epsilon^{2}\left(1-\eta^{2}\right)\right)\left(1-\eta^{2}\right) \frac{\partial}{\partial \eta} \\
\left.+\frac{\left(1-\epsilon^{2}\left(\xi^{2}+1\right)\right)\left(1-\epsilon^{2}\left(1-\eta^{2}\right)\right)\left(\xi^{2}+\eta^{2}\right)}{\left(1-\eta^{2}\right)\left(1+\xi^{2}\right)}\left(\frac{\partial}{\partial \phi}\right)^{2}\right]
\end{gathered}
$$

which is now separable. The $\phi$-dependence of its eigenfunctions is taken care of by factors $e^{i m \phi}$ with the integer azimuthal quantum number $m$. Separating the operator in $\xi$ and $\eta$ by making the ansatz $\Psi_{\xi}(\xi) \Psi_{\eta}(\eta) e^{i m \phi}$ for its eigenfunctions we obtain two equations, one for $\Psi_{\eta}$

$$
\begin{array}{r}
{\left[\frac{d}{d \eta}\left(1-\eta^{2}\right) \frac{d}{d \eta}-\frac{m^{2}}{1-\eta^{2}}+\frac{2 \epsilon^{2}\left(1-\eta^{2}\right) \eta}{1-\epsilon^{2}\left(1-\eta^{2}\right)} \frac{d}{d \eta}\right] \Psi_{\eta}} \\
-\frac{2 \omega^{2} / \omega_{1}^{2}}{1-\epsilon^{2}\left(1-\eta^{2}\right)} \Psi_{\eta}=-\beta \Psi_{\eta} .
\end{array}
$$

the other for $\Psi_{\xi}$. It turns out that both equations are identical if in the equation for $\Psi_{\eta}$ we substitute $i \xi$ for $\eta$, i.e. $\Psi_{\xi}(\xi) \equiv \Psi_{\eta}(i \xi)$. The solution for one coordinate is the analytic continuation of the solution of the other from the real to the imaginary axis. It is easy to check that the separation constant $\beta$ is just the eigenvalue of the operator $\hat{B}$ for the eigenfunction $\Psi_{\xi}(\xi) \Psi_{\eta}(\eta) \exp (i m \phi)$. To do this one needs to express $\hat{B}$ also in the elliptic coordinates:

$$
\begin{aligned}
\hat{B} & =\frac{1}{\epsilon^{2}\left(\xi^{2}+\eta^{2}\right)}\left\{-\frac{\partial}{\partial \xi}\left(1-\epsilon^{2}\left(\xi^{2}+1\right)\right)\left(\xi^{2}+1\right) \frac{\partial}{\partial \xi}\right. \\
& -\frac{\partial}{\partial \eta}\left(1-\epsilon^{2}\left(1-\eta^{2}\right)\right)\left(1-\eta^{2}\right) \frac{\partial}{\partial \eta} \\
& \left.+\frac{\xi^{2}+\eta^{2}}{\left(\xi^{2}+1\right)\left(1-\eta^{2}\right)} \frac{\partial^{2}}{\partial \phi^{2}}\right\} .
\end{aligned}
$$


The equations (5) contain $m$ only quadratically. Therefore the energy levels are the same for $\pm m$. Expanding $\Psi_{\eta}$ in terms of associated Legendre functions

$$
\Psi_{\eta}=\sum_{\ell=|m|}^{\infty} a_{\ell} P_{\ell}^{|m|}(\eta)
$$

we obtain from eq. (5) a second order recursion relation for the coefficients $a_{\ell}$ relating only even or only odd indices $\ell$. The eigenstates therefore have even and odd parity. The recursion relation itself is straightforward to obtain but lengthy and will not be written out here. The condition that (7) terminates at $\ell_{\max }=(|m|+n)$ quantizes the eigenvalue $\beta$

$$
\beta=(n+|m|)(n+|m|+3),
$$

which means that $\Psi_{\eta}$ becomes $\left(1-\eta^{2}\right)^{|m / 2|}$ times a polinomial of order $n$.

In the isotropic case $\epsilon=0$ the operator $\hat{B}$ can be diagonalized in spherical coordinates and its spectrum then is found as $\beta=\left(2 n_{r}+\ell\right)\left(2 n_{r}+\ell+3\right)$ with radial quantum number $n_{r}$ and angular quantum number $\ell$. Together with the connection of $\hat{G}$ and $\hat{B}$ for isotropic traps this gives the result of 16] for the spectrum in the isotropic case.

The eigenvalue condition for $\omega^{2}$ takes the form of the characteristic equation of a tridiagonal matrix of dimension $N=1+\operatorname{int}[n / 2]$, which can be symmetrized by a suitable similarity transformation with a given diagonal matrix. For fixed numbers $n,|m|$ we have $N$ different solutions for $\omega^{2}$, which we label by our third quantum number $j=0, \ldots$ int $[n / 2]$. In the isotropic case $\epsilon=0$ the quantum number $j$ can be expressed as $j=\operatorname{int}[(\ell-|m|) / 2]=\operatorname{int}[n / 2]-n_{r}$ as one finds by expressing the isotropic spectrum of [16] in terms of the new quantum numbers $n, j \leq \operatorname{int}[n / 2]$.

Calculating the first levels $\omega(n, j, m)$ we get with $\lambda=$ $\left(\omega_{0} / \omega_{z}\right)^{2}$

$$
\begin{aligned}
& \omega^{2}(0,0, m)=\omega_{0}^{2}|m| \\
& \omega^{2}(1,0, m)=\omega_{z}^{2}+\omega_{0}^{2}|m| \\
& \omega^{2}(2, j(=0,1), m)=\omega_{z}^{2}\left(\frac{3}{2}+2(|m|+1) \lambda\right. \\
& \left.\quad-\frac{(-1)^{j}}{2}\left(9-4(|m|+4) \lambda+4(|m|+2)^{2} \lambda^{2}\right)^{1 / 2}\right) \\
& \omega^{2}(3, j(=0,1), m)=\omega_{z}^{2}\left(\frac{7}{2}+2(|m|+1) \lambda\right. \\
& \left.\quad-\frac{(-1)^{j}}{2}\left(25+4(|m|-4) \lambda+4(|m|+2)^{2} \lambda^{2}\right)^{1 / 2}\right) .
\end{aligned}
$$

In the limit $\lambda^{-1} \rightarrow 0$, which is relevant for the experiments reported in [5], the mode frequencies for arbitrary integer $n \geq 2 j$ not to large can be expanded in the small parameter $\lambda^{-1}$ and are found as

$$
\omega^{2}(n, j, m)=\omega_{0}^{2}\left(|m|+2 j(j+|m|+1)+O\left(\lambda^{-1}\right)\right)
$$

Remarkably, the leading order term for $|m|, j$ not both vanishing is independent of $n$, i.e. the levels consist in this case of bands of closely spaced levels split only by small frequencies of order $\omega_{z}^{2} / \omega_{0}$.

Eq.(9) contains, as special cases, the particular solutions previously obtained by Stringari [16] (the $n=$ 0,$1 ; j=0,1$ modes for all $|m|$ and the two $n=2 ; j=$ 0,$1 ; m=0$ modes).

After our calculation had been completed and while this paper was being prepared a preprint became available [20], in which the mode frequencies (9) where also found by solving the wave equation directly via a polynomial ansatz, which can be shown to be equivalent to ours. This method works because, as we have shown above, no special boundary conditions except regularity need to be imposed on the wave function at the surface of the condensate. However, the deeper reason for the solvability of the equation, i.e. the additional conservation law, has not been identified in 20].

Because of the necessity to diagonalize $N$-dimensional matrices the analytical determination of the $\omega^{2}$ by the present method for $\lambda$ neither very small nor very large is possible up to $N=4$, even though the formulas for $N=3$ and $N=4$ are too cumbersome to be of much practical value. However, it is straightforward to diagonalize the matrices numerically and to prepare a list of the numerical values of the eigenvalues for a given ratio $\lambda$, e.g. for the experimental value $\lambda=1 / 8$. In table I we give such a list for the 43 lowest lying eigenvalues with $|m| \leq 2$ in units of $\omega_{0}$ for $\lambda=1 / 8$, together with their quantum numbers $m, n, j$. A numerical evaluation of the energy levels of the collective modes as a function of the scaling variable 16 $N a / d_{0}$ for $\lambda=1 / 8$ was recently reported in [9]. Here $N$ is the number of atoms and $d_{0}=\sqrt{\hbar / 2 M \omega_{0}}$ is a measure of the size of the ground state in the trap. The present results apply for $N a / d_{0} \rightarrow \infty$. Comparing our results with those in [9] we find that the rate of convergence to the asymptotic case $N a / d_{0} \rightarrow \infty$ depends on the quantum number $n$ and is much slower, e.g. for $n=6$ than for $n=2$. For $m=0$ we find 4 additional levels (with quantum numbers $n=5,7,8,9$ and $j=0$ ) below the highest $\mathrm{m}=0$ level considered in [9], where the motion of the levels was tracked from the free particle case to the strongly interacting case. The explanation can be that levels having rather high frequency in the free trap can move down into the considered low-energy regime as the parameter $N a / d_{0}$ is increased. Following the levels one can expect a number of avoided level crossings due to the non-integrability of the system at intermediate energies. We demonstrate the chaoticity of the corresponding classical system whose Hamiltonian

$$
\begin{aligned}
H(\boldsymbol{p}, \boldsymbol{x}) & =\sqrt{\epsilon_{H F}^{2}(\boldsymbol{p}, \boldsymbol{x})-K^{2}(\boldsymbol{x})}, \\
\epsilon_{H F}(\boldsymbol{p}, \boldsymbol{x}) & =\frac{\boldsymbol{p}^{2}}{2 M}+U(\boldsymbol{x})-\mu+2 K(\boldsymbol{x}) \\
K(\boldsymbol{x}) & =(\mu-U(\boldsymbol{x})) \Theta(\mu-U(\boldsymbol{x}))
\end{aligned}
$$


is the Bogoliubov quasiparticle energy in local density approximation 21] (Fig.1)

In conclusion we have demonstrated that the quasiparticle dynamics in axially symmetric traps is chaotic for energies comparable to the chemical potential, but, for energies much smaller than $\mu$ we found a third conserved variable $\hat{B}$ commuting with the wave operator $\hat{G}$ and axial angular momentum $\hat{L}_{z}$. As a consequence the wave equation in this regime is proven to be integrable which explains why some solutions have already been found in the literature. We have separated the wave equations in elliptical coordinates, reduced the eigenvalue problem to the diagonalization of finite dimensional matrices, and exhibited three integer quantum numbers $m, n, j$ in the ranges $-\infty \leq m \leq+\infty, 0 \leq n<\infty, 0 \leq j \leq \operatorname{int}[n / 2]$ labelling all modes and their frequencies for the experimentally realized case $\omega_{z}^{2} / \omega_{0}^{2}=8$. As a final remark we mention that we have found even the fully anisotropic case, where the axial angular momentum is no longer a good quantum number, to be integrable [22]. Two new conserved quantities can be identified in that case which reduce to $\hat{L}_{z}$ and $\hat{B}$ in the axially symmetric limit.

\section{ACKNOWLEDGEMENTS}

One of us (R.G.) acknowledges discussions with J. Ruostekoski. This work has been supported by the project of the Hungarian Academy of Sciences and the Deutsche Forschungsgemeinschaft under grant No. 95. M.F. gratefully acknowledges support by the German-Hungarian Scientific and Technological Cooperation under Project 62. R.G. and M. F. wishes to acknowledge support by the Deutsche Forschungsgemeinschaft through the Sonderforschungsbereich 237 "Unordnung und große Fluktuationen". Two of us (A.Cs,P.Sz) would like to acknowledge support by the Hungarian Academy of Sciences under grant No. AKP 96-12/12. The work has been partially supported by the Hungarian National Scientific Research Foundation under grant Nos. OTKA T017493 and F020094.

[1] M. H. Anderson et al., Science 269, 198 (1995).

[2] K.B. Davis et al., Phys. Rev. Lett. 75, 3969 (1995).

[3] C.C. Bradley et al., Phys. Rev. Lett. 75, 1687 (1995).

[4] D. S. Jin et al., Phys. Rev. Lett. 77, 420 (1996).

[5] M.O. Mewes et al., Phys. Rev. Lett. 77, 416 (1996).

[6] D. S. Jin et al., Phys. Rev. Lett. 78, 764 (1997).

[7] M. R. Andrews et al., Science 27384 (1996).

[8] M. Edwards etal., Phys. Rev. Lett. 77, 1671 (1996).

[9] L. You et al., Phys. Rev. A55, R1581 (1997).

[10] A. L. Fetter, Phys. Rev. A53, 4245 (1996)
[11] V. M. Pérez-García et al., Phys. Rev. Lett 77, 1520 (1996).

[12] Y. Castin and R. Dum, Phys. Rev. Lett. 77, 5315 (1996).

[13] Yu Kagan et al., Phys. Rev. A55, R18 (1997).

[14] A. Csordás et al.,unpublished cond-mat/9705133.

[15] K. G. Singh and D. S. Roksahr, Phys. Rev. Lett. 77, 1667 (1996).

[16] S. Stringari, Phys. Rev. Lett. 77, 2360 (1996).

[17] A. L. Fetter, Phys. Rev. A53, 4245 (1996); W.-C. Wu and A. Griffin, Phys. Rev. A54, 4204 (1996); A. L. Fetter, D. L. Feder, unpublished (cond-mat/9704173); A. L. Fetter, D. Rokshar, unpublished (cond-mat/9704234).

[18] G. Baym and C. J. Pethick, Phys. Rev. Lett. 76, 6 (1996).

[19] Ph. M. Morse and H. Feshbach, Methods of Theoretical Physics, Part I, McGraw Hill (New York 1953).

[20] P. Öhberg et al., unpublished (physics/9705006).

[21] S. Giorgini et al., Phys. Rev. A54, 4633 (1996).

[22] A. Csordás et al., to be published.

FIG. 1. Poincaré sections of the classical dynamics in cylindrical coordinates $\rho, z, \phi$ after the elimination of the conserved axial angular momentum and the azimuthal angle $\phi$ for energy $E / \mu=1$. The cut is taken at $z=0$ and diplayed in the scaled variables $\rho, p_{\rho}$ measured in units of $\sqrt{2 \mu / m \omega_{0}^{2}}$ and $\sqrt{2 m \mu}$, respectively. The anisotropy is chosen as $\omega_{z} / \omega_{0}=\sqrt{8}$, the angular momentum was fixed as $\omega_{0} L_{z} / E=0.2$.

\begin{tabular}{rllll}
\hline \hline $\mathrm{n}$ & $\mathrm{j}$ & $m=0$ & $m=1$ & $m=2$ \\
\hline 0 & 0 & 0.00000000 & 1.00000000 & 1.41421356 \\
2 & 0 & 1.79712837 & 2.31729458 & 2.72341601 \\
1 & 0 & 2.82842712 & 3.00000000 & 3.16227766 \\
4 & 0 & 2.91193010 & 3.36537641 & 3.73131058 \\
3 & 0 & 3.27302589 & 3.51832107 & 3.74165739 \\
5 & 0 & 3.82657318 & 4.09894174 & 4.34324821 \\
6 & 0 & 3.84099626 & 4.21532329 & 4.52279302 \\
7 & 0 & 4.40748368 & 4.67921086 & 4.92221757 \\
8 & 0 & 4.59081095 & 4.89814704 & 5.16010207 \\
9 & 0 & 4.97105460 & 5.22935896 & 5.46144995 \\
2 & 1 & 4.97697997 & 5.16044047 & 5.34630763 \\
10 & 0 & 5.20648119 & 5.47081354 & 5.70417681 \\
4 & 1 & 5.45030761 & 5.74456265 & $(6.04142431)$ \\
11 & 0 & 5.49925114 & 5.74053518 & 5.95925695 \\
12 & 0 & 5.73868980 & 5.97616320 & $(6.19077758)$ \\
\hline \hline
\end{tabular}

TABLE I. The 43 lowest levels labeled by the quantum numbers $|m| \leq 2, n, j$ for $\left(\omega_{z} / \omega_{0}\right)^{2}=8$ 


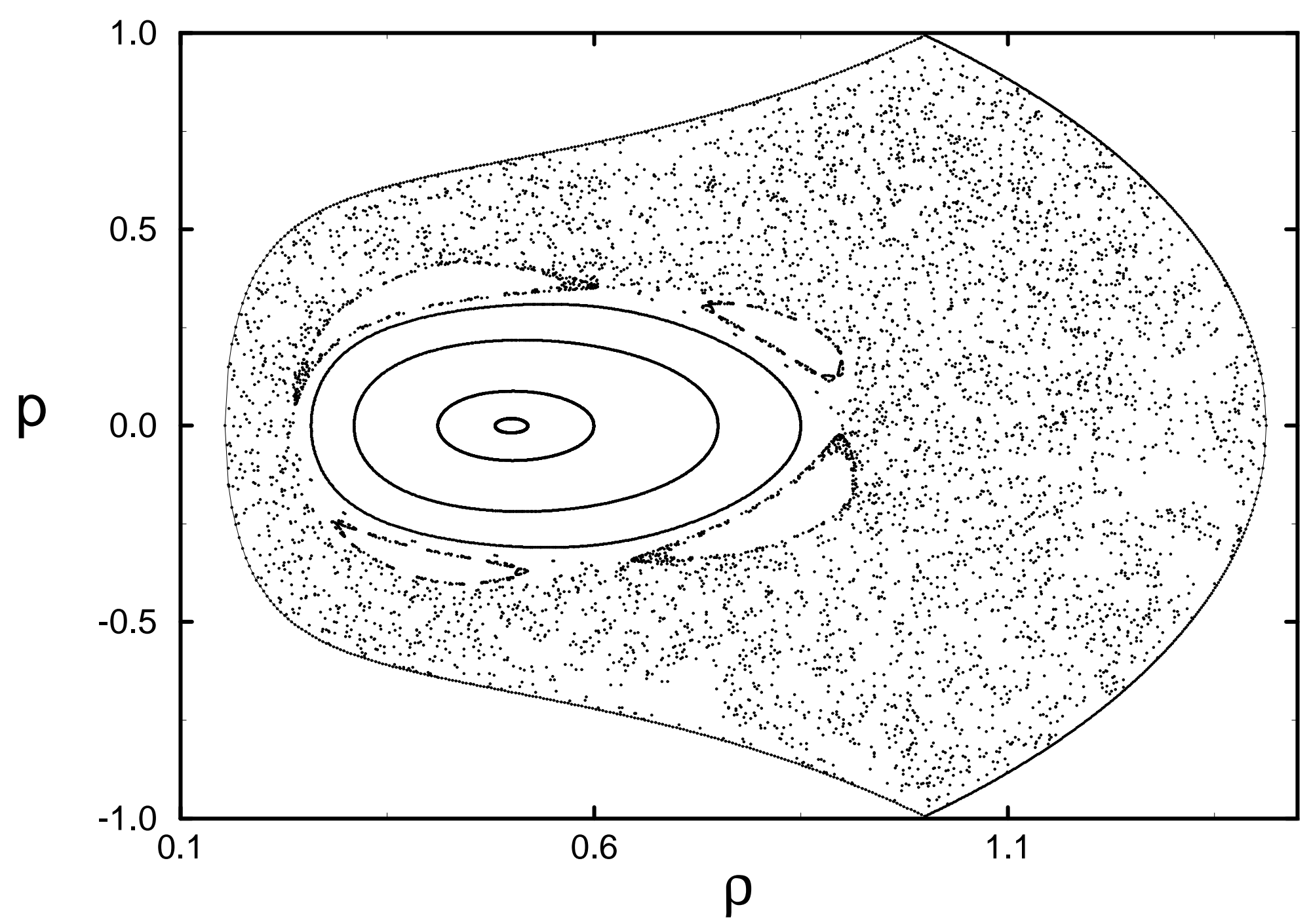

